International Journal of Pure and Applied Mathematics

Volume 85 No. 5 2013, 907-923

ISSN: 1311-8080 (printed version); ISSN: 1314-3395 (on-line version)

url: http://www.ijpam.eu

doi: http://dx.doi.org/10.12732/ijpam.v85i5.9

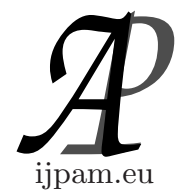

\title{
CONTINUOUS FOURTH DERIVATIVE METHOD FOR THIRD ORDER BOUNDARY VALUE PROBLEMS
}

\author{
R.K. Sahi ${ }^{1}$, S.N. Jator ${ }^{2}$, N.A. Khan ${ }^{3}$ \\ ${ }^{1,2}$ Department of Mathematics and Statistics \\ Austin Peay State University \\ Clarksville, TN 37044, USA \\ ${ }^{3}$ Department of Mathematics \\ The University of North Texas at Dallas \\ Dallas, TX 75044, USA
}

\begin{abstract}
A fourth derivative method (FDM) with continuous coefficients is derived and used to obtain main and additional methods which are used to solve third order boundary value problems (TOBVPs) such as the Blasius, the Falkner-Skan, and the sandwich beam problems which frequently occur in engineering. The convergence analysis of the method is discussed. Numerical experiments are performed to show speed and accuracy advantages.
\end{abstract}

AMS Subject Classification: 65L05, 65L06, 65L10, 65L12

Key Words: third order boundary value problems, fourth derivative method, sandwich problem, Falkner-Skan and Blasius equations

\section{Introduction}

In the last three decades, third order boundary value problems (BVP's) have gained a lot of attention in the literature. These kind of BVP's have applications in the field of engineering and science, especially in control theory, and biological sciences. For instance, draining and coating flow problems, see Tuck and Schwartz [21]; laminar boundary layer and sandwich beam problems, see

Received: March 26, 2013

(C) 2013 Academic Publications, Ltd.

${ }^{\S}$ Correspondence author url: www.acadpubl.eu 
[18], and [20]; and in fluid dynamics, see Valarmathi and Ramanujam [22].

In the past decades, tremendous attention has been focused on developing methods for the solution of $y^{\prime \prime}=f\left(x, y, y^{\prime}, y^{\prime \prime}\right)$ subject to boundary conditions (see Awoyemi[2], Jator [6], [7] ). Many methods like standard finite difference (SFDs), splines ( Khan and Aziz [12]),non-polynomial splines (Siraj-ul-Islam and Tirmizi [19]), high order difference methods (Salama and Mansour [17]) are used in solving third order BVP's. Most of these methods are solved by first reducing a higher order ordinary differential equation (ODE) to an equivalent system of first-order ODE's which takes a lot of human effort and computer time as discussed in Awoyemi[2].

In this paper, we consider the general third-order BVP

$$
D y \equiv y^{\prime \prime \prime}=f\left(x, y, y^{\prime}, y^{\prime \prime}\right)
$$

subject to any of the following boundary conditions:

$$
\begin{aligned}
& y(a)=y_{0}, y^{\prime}(a)=\delta_{0}, y(b)=y_{N} \\
& y(a)=y_{0}, y^{\prime}(a)=\delta_{0}, y^{\prime}(b)=y_{M} \\
& y(a)=y_{0}, y^{\prime}(b)=y_{M}, y(c)=\gamma_{\frac{1}{2}}, \text { where } c=\left(\frac{a+b}{2}\right)
\end{aligned}
$$

where $a, b, c, \delta_{0}, y_{0}, y_{N}, y_{M}$ are constants, $f$ is a continuous function and satisfies a Lipschitz condition as given in [4]. Keller [10] has given the theorem and the proof of the general conditions which ensure that the solution to (1) will exist and be unique.

Using multistep collocation a continuous FDM is derived, see Lie and Norsett [13], Atkinson [1], and Onumanyi et al [15]. The continuous representation generates the basic FDM and $k-1$ additional methods which are combined and used to simultaneously produce approximations $y_{j}$, for $j=1, \ldots, N-1$ to the solution of (1) at points $x_{j}$ for $j=1, \ldots, N-1$ on $\pi_{N}: a=x_{0}<x_{1}<\ldots<x_{N}=b$

The basic and auxiliary methods are obtained from the same continuous scheme and are of the same order, hence, possible errors which are due to auxiliary methods of lower order are avoided as the integration proceeds on the entire interval.

The paper is organized as follows. In section two, we derive an approximation $U(x)$ for $y(x)$ which is used to obtain the main and additional FDMs. The convergence analysis of the method is also given in Section 2. Section 3 is devoted to the computational aspects and an algorithm equipped with an automatic error estimate based on the double mesh principle. Numerical examples are given in Section 4 to show speed and accuracy advantages. Finally, the conclusion of the paper is discussed in Section 5. 


\section{Construction of the CFDMs with FDMs as by Products}

In this section, we construct the CFDMs with FDMs as by products. Thus, on the interval $\left[x_{n}, x_{n+k}\right]$, we assume that the exact solution $y(x)$ and its first and second derivatives are locally represented by the continuous methods

$$
\begin{gathered}
U_{k}(x)=\sum_{j=0}^{k-1} \alpha_{j}(x) y_{n+j}+h^{3} \sum_{j=0}^{k} \beta_{j}(x) f_{n+j}+h^{4} \sum_{j=0}^{k} \gamma_{j}(x) g_{n+j} \\
\left\{\begin{array}{l}
U_{k}^{\prime}(x)=\frac{d}{d x}(U(x)) \\
U_{k}^{\prime \prime}(x)=\frac{d^{2}}{d x^{2}}(U(x))
\end{array}\right.
\end{gathered}
$$

where $\alpha_{j}(x), \beta_{j}(x), \gamma_{j}(x)$ are continuous coefficients, and $m>0$ is an integer.

We assume that $y_{n+j}=U_{k}\left(x_{n}+j h\right)$ is the numerical approximation to the analytical solution $y\left(x_{n+j}\right), y_{n+j}^{\prime}=U_{k}^{\prime}\left(x_{n}+j h\right)$ is an approximation to $y^{\prime}\left(x_{n+j}\right)$, $y_{n+j}^{\prime \prime}=U_{k}^{\prime \prime}\left(x_{n}+j h\right)$ is an approximation to $y^{\prime \prime}\left(x_{n+j}\right), f_{n+j}=U_{k}^{\prime \prime \prime}\left(x_{n}+j h\right)$, and $g_{n+j}=U_{k}^{\prime \prime \prime \prime}\left(x_{n}+j h\right)$. We also note that $f_{n+j}=f\left(x_{n+j}, y_{n+j}, y_{n+j}^{\prime}, y_{n+j}^{\prime \prime}\right)$, $g_{n+j}=\left.\frac{d f\left(x, y(x), y^{\prime}(x), y^{\prime \prime}(x)\right)}{d x}\right|_{y_{n+j}, y_{n+j}^{\prime}, y_{n+j}^{\prime \prime}} ^{x_{n+j}}, j=0, \ldots, 3$.

The continuous method (2) and its first and second derivatives (3) are piecewise continuous on $[a, b]$ and defined for all $x \in[a, b]$. That is, $U_{k}(x), U_{k}^{\prime}(x), U_{k}^{\prime \prime}(x)$ are defined such that $\left.U_{k}(x)=y(x)+O\left(h^{11}\right)\right), U_{k}^{\prime}(x)=\frac{d}{d x}\left(y(x)+O\left(h^{11}\right)\right), U_{k}^{\prime \prime}(x)=$ $\frac{d^{2}}{d x^{2}}\left(y(x)+O\left(h^{11}\right)\right), x \epsilon\left(x_{n}, x_{n+3}\right)$. The polynomials $\left\{U_{0}(x), U_{3}(x), \ldots, U_{N-3}(x)\right\}$, $\left\{U_{0}^{\prime}(x), U_{3}^{\prime}(x), \ldots, U_{N-3}^{\prime}(x)\right\},\left\{U_{0}^{\prime \prime}(x), U_{3}^{\prime \prime}(x), \ldots, U_{N-3}^{\prime \prime}(x)\right\}$, then define piecewise polynomials $U(x), U^{\prime}(x)$, and $U^{\prime \prime}(x)$ which are also continuous on $[a, b]$. Hence, (2) and (3) have the ability to provide a continuous solution on $[a, b]$ with a uniform accuracy comparable to that obtained at the grid points (see Onumanyi et al. [15]) and can also be used to produce additional discrete methods (see Onumanyi et al. [14]). In what follows, we state the theorem that facilitates the construction of the CFDMs (2) and (3).

Theorem 2.1. Let the following conditions be satisfied

$$
\begin{gathered}
U_{k}\left(x_{n+j}\right)=y_{n+j}, j=0,1,2 \\
U_{k}^{\prime \prime \prime}\left(x_{n+j}\right)=f_{n+j}, \quad U_{k}^{\prime \prime \prime \prime}\left(x_{n+j}\right)=g_{n+j}, j=0, \ldots, 3,
\end{gathered}
$$

then, the continuous representations (2) and (3) are equivalent to the following:

$$
U_{k}(x)=V^{T}\left(W^{-1}\right)^{T} P(x)
$$




$$
\left\{\begin{array}{l}
U_{k}^{\prime}(x)=\frac{d}{d x}\left(V^{T}\left(W^{-1}\right)^{T} P(x)\right) \\
U_{k}^{\prime \prime}(x)=\frac{d^{2}}{d x^{2}}\left(V^{T}\left(W^{-1}\right)^{T} P(x)\right)
\end{array}\right.
$$

where $W$ is given as

$$
\begin{gathered}
W=\left(\begin{array}{llc}
P_{0}\left(x_{n}\right) & \cdots & P_{10}\left(x_{n}\right) \\
P_{0}\left(x_{n+1}\right) & \cdots & P_{10}\left(x_{n+1}\right) \\
P_{0}\left(x_{n+2}\right) & \cdots & P_{10}\left(x_{n+2}\right) \\
P_{0}^{\prime \prime \prime}\left(x_{n}\right) & \cdots & P_{10}^{\prime \prime \prime}\left(x_{n}\right) \\
P_{0}^{\prime \prime \prime}\left(x_{n+1}\right) & \cdots & P_{10}^{\prime \prime \prime}\left(x_{n+1}\right) \\
P_{0}^{\prime \prime \prime}\left(x_{n+2}\right) & \cdots & P_{10}^{\prime \prime \prime}\left(x_{n+2}\right) \\
P_{0}^{\prime \prime \prime}\left(x_{n+3}\right) & \cdots & P_{10}^{\prime \prime \prime}\left(x_{n+3}\right) \\
P_{0}^{\prime \prime \prime \prime}\left(x_{n}\right) & \cdots & P_{10}^{\prime \prime \prime \prime}\left(x_{n}\right) \\
P_{0, \prime \prime}^{\prime \prime \prime}\left(x_{n+1}\right) & \cdots & P_{10}^{\prime \prime \prime}\left(x_{n+1}\right) \\
P_{0, \prime \prime}^{\prime \prime \prime}\left(x_{n+2}\right) & \cdots & P_{10}^{\prime \prime \prime}\left(x_{n+2}\right) \\
P_{0}^{\prime \prime \prime}\left(x_{n+3}\right) & \cdots & P_{10}^{\prime \prime \prime \prime}\left(x_{n+3}\right)
\end{array}\right), \\
V=\left(y_{n}, y_{n+1}, y_{n+2}, f_{n}, f_{n+1}, f_{n+2}, f_{n+3}, g_{n}, g_{n+1}, g_{n+2}, g_{n+3}\right)^{T}, \\
P(x)=\left(P_{0}(x), P_{1}(x), \ldots, P_{10}(x)\right)^{T} .
\end{gathered}
$$

We note that $T$ denotes the transpose and $P_{j}(x)=x^{j}, j=0, \ldots, 10$ are basis functions.

Proof. The proof is the same as in Jator et al. [9].

Remark 2.2. We emphasize that the continuous methods (2) and (3) which are equivalent to the forms (6) and (7) are used to produce the main and additional methods which are combined and simultaneously applied to provide all approximations on the entire interval for boundary value problems of the form $y^{\prime \prime}=f\left(x, y, y^{\prime}, y^{\prime \prime}\right)$.

Remark 2.3. The continuous methods (2) and (3) are obtained by solving a system of 11 equations resulting from conditions (4) and (5) given in theorem 2.1 .

Coefficients of the CFDMs (2). In order to simplify the coefficients of (2), we introduce the scale variable $t=\frac{x-x_{n+2}}{h}$ to express the coefficients of the CFDM as follows:

$$
\alpha_{0}(t)=\frac{1}{2}\left(t+t^{2}\right) ; \alpha_{1}(t)=\left(-2 t-t^{2}\right) ; \quad \alpha_{2}(t)=\frac{1}{2}\left(2+3 t+t^{2}\right)
$$




$$
\begin{aligned}
& \beta_{0}(t)=\frac{1}{544320}\left(6950 t+7891 t^{2}+2100 t^{5}+462 t^{6}-1200 t^{7}-330 t^{8}+250 t^{9}+77 t^{10}\right) \\
& \beta_{1}(t)=\frac{1}{20160}\left(4268 t+4886 t^{2}+672 t^{5}-168 t^{6}-240 t^{7}+15 t^{8}+40 t^{9}+7 t^{10}\right) \\
& \beta_{2}(t)=\frac{1}{20160}\left(2094 t+4759 t^{2}+3360 t^{3}-924 t^{5}-42 t^{6}+240 t^{7}+30 t^{8}-30 t^{9}-7 t^{10}\right) \\
& \beta_{3}(t)=\frac{1}{544320}\left(2716 t+3854 t^{2}+4704 t^{5}+5208 t^{6}+1200 t^{7}-885 t^{8}-520 t^{9}-77 t^{10}\right) \\
& \gamma_{0}(t)=\frac{1}{181440}\left(430 t+503 t^{2}+168 t^{5}+42 t^{6}-96 t^{7}-30 t^{8}+20 t^{9}+7 t^{10}\right) \\
& \gamma_{1}(t)=+\frac{1}{20160}\left(228 t+434 t^{2}+336 t^{5}-168 t^{7}-15 t^{8}+30 t^{9}+7 t^{10}\right) \\
& \gamma_{2}(t)=\frac{1}{20160}\left(-610 t-1009 t^{2}+840 t^{4}+336 t^{5}-294 t^{6}-192 t^{7}+30 t^{8}\right. \\
&\left.+40 t^{9}+7 t^{10}\right) \\
& \gamma_{3}(t)=+\frac{1}{181440}\left(-212 t-298 t^{2}-336 t^{5}-336 t^{6}-24 t^{7}+105 t^{8}+50 t^{9}\right. \\
&\left.+7 t^{10}\right)
\end{aligned}
$$

FDMs. The following main methods are obtained by evaluating (2) and (3) at $x=x_{n+3}$.

$$
\begin{aligned}
y_{n+3}-3 y_{n+2}+3 y_{n+1}-y_{n} & =\frac{h^{3}}{168}\left(5 f_{n}+79 f_{n+1}+79 f_{n+2}+5 f_{n+3}\right) \\
+ & \frac{h^{4}}{5040}\left(29 g_{n}+213 g_{n+1}-213 g_{n+2}-29 g_{n+3}\right),
\end{aligned}
$$

$$
\begin{aligned}
& h y_{n+3}^{\prime}- \frac{3}{2} y_{n}+4 y_{n+1}-\frac{5}{2} y_{n+2} \\
&=\frac{h^{3}}{90720}\left(4664 f_{n}+68679 f_{n+1}+82800 f_{n+2}+10177 f_{n+3}\right) \\
& \quad+\frac{h^{4}}{30240}\left(311 g_{n}+2730 g_{n+1}-39 g_{n+2}-552 g_{n+3}\right),
\end{aligned}
$$

$$
\begin{aligned}
& h^{2} y_{n+3}^{\prime \prime}-y_{n}+2 y_{n+1}-y_{n+2}=\frac{h^{3}}{272160}\left(13846 f_{n}+167967 f_{n+1}+255258 f_{n+2}\right. \\
& \left.+107249 f_{n+3}\right)+\frac{h^{4}}{90720}\left(992 g_{n}+11169 g_{n+1}+13896 g_{n+2}-4147 g_{n+3}\right) .
\end{aligned}
$$

The following additional methods are obtained by evaluating (3) at $\{x=$ 
$\left.x_{0}, x_{1}, x_{2}\right\}$.

$$
\left\{\begin{aligned}
-2 y_{1}=-h y_{0}^{\prime}-\frac{3}{2} y_{0}-\frac{1}{2} y_{2}+\frac{h^{3}}{90720}\left(6127 f_{0}+18810 f_{1}+4689 f_{2}+614 f_{3}\right) \\
+\frac{h^{4}}{30240}\left(291 g_{0}-1878 g_{1}-813 g_{2}-50 g_{3}\right) \\
-y_{2}=-h^{2} y_{0}^{\prime \prime}+y_{0}-2 y_{1}+\frac{h^{3}}{272160}\left(-99149 f_{0}-127278 f_{1}-39987 f_{2}-5746 f_{3}\right) \\
\quad+\frac{h^{4}}{18144}\left(-725 g_{0}+3546 g_{1}+1467 g_{2}+94 g_{3}\right) \\
h y_{1}^{\prime}=-\frac{1}{2} y_{0}+\frac{1}{2} y_{2}+\frac{h^{3}}{272160}\left(-2692 f_{0}-35721 f_{1}-6372 f_{2}-575 f_{3}\right) \\
\quad+\frac{h^{4}}{90720}\left(-155 g_{0}+828 g_{1}+891 g_{2}+46 g_{3}\right) \\
h y_{2}^{\prime}=\frac{1}{2} y_{0}-2 y_{1}+\frac{3}{2} y_{2}+\frac{h^{3}}{272160}\left(3475 f_{0}+57618 f_{1}+28269 f_{2}+1358 f_{3}\right) \\
\quad+\frac{h^{4}}{90720}\left(215 g_{0}+1026 g_{1}-2745 g_{2}-106 g_{3}\right) \\
h^{2} y_{1}^{\prime \prime}=y_{0}-2 y_{1}+y_{2}+\frac{h^{3}}{272160}\left(4246 f_{0}-513 f_{1}-3942 f_{2}+209 f_{3}\right) \\
\quad+\frac{h^{4}}{90720}\left(224 g_{0}-5247 g_{1}+72 g_{2}-19 g_{3}\right) \\
h^{2} y_{2}^{\prime \prime}=y_{0}-2 y_{1}+y_{2}+\frac{h^{3}}{272160}\left(7891 f_{0}+131922 f_{1}+128493 f_{2}+3854 f_{3}\right) \\
\quad+\frac{h^{4}}{90720}\left(503 g_{0}+3906 g_{1}-9081 g_{2}-298 g_{3}\right)
\end{aligned}\right.
$$

\section{Convergence Analysis}

Local truncation error and order. The local truncation errors associated with the discrete fourth derivative method (FDM) main methods (8)-(10) are given by $O\left(h^{12}\right)$,

$\tau_{i}=\frac{47}{8467200} h^{11} y^{(11)}\left(x_{i}+\theta_{i}\right)+O\left(h^{12}\right), \quad h \tau_{i}^{\prime}=\frac{6641}{558835200} h^{11} y^{(11)}\left(x_{i}+\theta_{i}\right)+$

$h^{2} \tau_{i}^{\prime \prime}=\frac{29}{1693440} h^{11} y^{(11)}\left(x_{i}+\theta_{i}\right)+O\left(h^{12}\right), \quad i=3, \ldots, N, \quad\left|\theta_{i}\right| \leq 1$.

The local truncation errors associated with the first, second and third additional methods in (11) are given by

$$
\begin{aligned}
& \tau_{1}=\frac{71}{19958400} h^{11} y^{(11)}(\xi)+O\left(h^{12}\right), h \tau_{1}=-\frac{59}{62092800} h^{11} y^{(11)}(\xi)+O\left(h^{12}\right), h^{2} \tau_{1}^{\prime \prime}= \\
& \frac{17}{9313920} h^{11} y^{(11)}(\xi)+O\left(h^{12}\right), \quad x_{0} \leq \xi \leq x_{1} .
\end{aligned}
$$

Similarly, the local truncation errors associated with the fourth, fifth and sixth additional methods in (11) are given by

$\tau_{2}=-\frac{1}{86400} h^{11} y^{(11)}(\xi)+O\left(h^{12}\right), h \tau_{2}=\frac{19}{25401600} h^{11} y^{(11)}(\xi)+O\left(h^{12}\right), h^{2} \tau_{2}^{\prime \prime}=$ $\frac{61}{12700800} h^{11} y^{(11)}(\xi)+O\left(h^{12}\right), \quad x_{1} \leq \xi \leq x_{2}$.

Convergence. In order to show that the FDM converges, the methods (8), (9), (10), and (11) can be compactly written in matrix form by introducing the 
following notations. Let $A$ be a $3 N \times 3 N$ matrix defined by

$$
A=\left[\begin{array}{lll}
A_{11} & A_{12} & A_{13} \\
A_{21} & A_{22} & A_{23} \\
A_{31} & A_{32} & A_{33}
\end{array}\right],
$$

where the elements of $\mathrm{A}$ are $N \times N$ matrices given as

$$
\begin{aligned}
& A_{11}=\left[\begin{array}{ccccccccc}
-2 & 1 / 2 & 0 & 0 & 0 & 0 & \cdots & 0 & 0 \\
2 & -1 & 0 & 0 & 0 & 0 & \cdots & 0 & 0 \\
3 & -3 & 1 & 0 & 0 & 0 & \cdots & 0 & 0 \\
-1 & 3 & -3 & 1 & 0 & 0 & \cdots & 0 & 0 \\
0 & -1 & 3 & -3 & 1 & 0 & \cdots & 0 & 0 \\
\vdots & & & & \ddots & & & \vdots & \vdots \\
0 & 0 & 0 & \cdots & -1 & 3 & -3 & 1 & 0 \\
0 & 0 & 0 & \cdots & 0 & -1 & 3 & -3 & 1
\end{array}\right] \\
& A_{31}=\left[\begin{array}{cccccccc}
2 & -1 & 0 & 0 & 0 & \cdots & 0 & 0 \\
2 & -1 & 0 & 0 & 0 & \cdots & 0 & 0 \\
2 & -1 & 0 & 0 & 0 & \cdots & 0 & 0 \\
-1 & 2 & -1 & 0 & 0 & \cdots & 0 & 0 \\
0 & -1 & 2 & -1 & 0 & \cdots & 0 & 0 \\
\vdots & & & \ddots & & & \vdots & \vdots \\
0 & 0 & \cdots & -1 & 2 & -1 & 0 & 0 \\
0 & 0 & \cdots & 0 & -1 & 2 & -1 & 0
\end{array}\right] \\
& A_{21}=\left[\begin{array}{cccccccc}
0 & -1 / 2 & 0 & 0 & 0 & \ldots & 0 & 0 \\
2 & -3 / 2 & 0 & 0 & 0 & \ldots & 0 & 0 \\
4 & -5 / 2 & 0 & 0 & 0 & \ldots & 0 & 0 \\
-3 / 2 & 4 & -5 / 2 & 0 & 0 & \ldots & 0 & 0 \\
0 & -3 / 2 & 4 & -5 / 2 & 0 & \ldots & 0 & 0 \\
\vdots & & & \ddots & & & \vdots & \vdots \\
0 & 0 & \ldots & -3 / 2 & 4 & -5 / 2 & 0 \\
0 & 0 & \ldots & 0 & -3 / 2 & 4 & -5 / 2
\end{array}\right] \\
& A_{12}=A_{13}=A_{32}=\left[\begin{array}{ccccc}
0 & 0 & 0 & \cdots & 0 \\
0 & 0 & 0 & \cdots & 0 \\
0 & 0 & 0 & \cdots & 0 \\
\vdots & & & \ddots & \vdots \\
0 & 0 & 0 & \cdots & 0
\end{array}\right]
\end{aligned}
$$




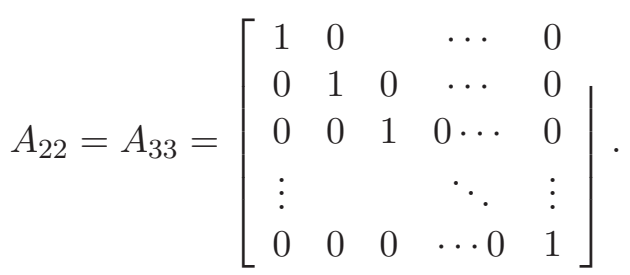

Similarly, let $B$ be a $3 N \times 3 N$ matrix defined by

$$
B=\left[\begin{array}{lll}
B_{11} & B_{12} & B_{13} \\
B_{21} & B_{22} & B_{23} \\
B_{31} & B_{32} & B_{33}
\end{array}\right],
$$

where the elements of $\mathrm{B}$ are $N \times N$ matrices given as

$$
\begin{aligned}
& B_{11}=h^{3}\left[\begin{array}{ccccccc}
\frac{18810}{90720} & \frac{4689}{90720} & \frac{614}{90720} & 0 & 0 & \cdots & 0 \\
\frac{-127278}{272160} & \frac{-39987}{272160} & \frac{-5746}{272160} & 0 & 0 & \cdots & 0 \\
\frac{79}{168} & \frac{79}{168} & \frac{5}{168} & 0 & 0 & \cdots & 0 \\
5 & \frac{79}{168} & \frac{79}{168} & \frac{5}{168} & 0 & \cdots & 0 \\
0 & 5 & \frac{79}{168} & \frac{79}{168} & \frac{5}{168} & \cdots & 0 \\
\vdots & & \ddots & & & \ldots & \vdots \\
0 & \ldots & 5 & \frac{79}{168} & \frac{79}{168} & \frac{5}{168} & 0 \\
0 & \ldots & 0 & 5 & \frac{79}{168} & \frac{79}{168} & \frac{5}{168}
\end{array}\right], \\
& B_{12}=h^{3}\left[\begin{array}{ccccccc}
\frac{-1878}{30240} & \frac{-813}{30240} & \frac{-50}{30240} & 0 & 0 & \ldots & 0 \\
\frac{1026}{30240} & \frac{-2745}{30240} & \frac{-106}{30240} & 0 & 0 & \ldots & 0 \\
\frac{213}{5040} & \frac{-213}{5040} & \frac{-29}{5040} & 0 & 0 & \ldots & 0 \\
\frac{29}{5040} & \frac{213}{5040} & \frac{-213}{5040} & \frac{-29}{5040} & 0 & \ldots & 0 \\
0 & \frac{29}{5040} & \frac{213}{5040} & \frac{-213}{5040} & \frac{-29}{5040} & \ldots & 0 \\
\vdots & & \ddots & & \vdots & \ldots & \vdots \\
0 & \ldots & \frac{29}{5040} & \frac{213}{5040} & \frac{-213}{5040} & \frac{-29}{5040} & 0 \\
0 & \ldots & 0 & \frac{29}{5040} & \frac{213}{5040} & \frac{-213}{5040} & \frac{-29}{5040}
\end{array}\right] \text {, } \\
& B_{21}=h^{3}\left[\begin{array}{ccccccc}
\frac{-35721}{272160} & \frac{-6372}{272160} & \frac{-575}{272160} & 0 & 0 & \ldots & 0 \\
\frac{57618}{272160} & \frac{28269}{272160} & \frac{1358}{272160} & 0 & 0 & \ldots & 0 \\
\frac{68679}{90720} & \frac{82800}{90720} & \frac{10177}{90720} & 0 & 0 & \ldots & 0 \\
\frac{4664}{90720} & \frac{68679}{90720} & \frac{82800}{90720} & \frac{10177}{90720} & 0 & \ldots & 0 \\
0 & \frac{4664}{90720} & \frac{68679}{90720} & \frac{82800}{90720} & \frac{10177}{90720} & \ldots & 0 \\
\vdots & & \ddots & & & \ldots & \vdots \\
0 & \ldots & \frac{4664}{90720} & \frac{68679}{90720} & \frac{82800}{90720} & \frac{10177}{90720} & 0 \\
0 & \ldots & 0 & \frac{4664}{90720} & \frac{68679}{90720} & \frac{82800}{90720} & \frac{10177}{90720}
\end{array}\right],
\end{aligned}
$$




$$
B_{22}=h^{3}\left[\begin{array}{ccccccc}
\frac{828}{90720} & \frac{891}{90720} & \frac{46}{90720} & 0 & 0 & \ldots & 0 \\
\frac{1026}{90720} & \frac{-2745}{90720} & \frac{-106}{90720} & 0 & 0 & \ldots & 0 \\
\frac{2730}{30240} & \frac{-39}{30240} & \frac{-552}{30240} & 0 & 0 & \ldots & 0 \\
\frac{311}{30240} & \frac{2730}{30240} & \frac{-39}{30240} & \frac{-552}{30240} & 0 & \ldots & 0 \\
0 & & & & & \ldots & 0 \\
\vdots & & \ddots & & \vdots & \ldots & \vdots \\
0 & \ldots & \frac{311}{30240} & \frac{2730}{30240} & \frac{-39}{30240} & \frac{-552}{30240} & 0 \\
0 & \ldots & 0 & \frac{311}{30240} & \frac{2730}{30240} & \frac{-39}{30240} & \frac{-552}{30240}
\end{array}\right],
$$

We further define the following vectors:

$$
\begin{gathered}
Y=\left(y\left(x_{1}\right), \ldots, y\left(x_{N}\right), h y^{\prime}\left(x_{1}\right), \ldots, h y^{\prime}\left(x_{N}\right), h^{2} y^{\prime \prime}\left(x_{1}\right), \ldots, h^{2} y^{\prime \prime}\left(x_{N}\right)\right)^{T}, \\
\bar{Y}=\left(y_{1}, \ldots, y_{N}, h y_{1}^{\prime}, \ldots, h y_{N}^{\prime}, h^{2} y_{1}^{\prime \prime}, \ldots, h^{2} y_{N}^{\prime \prime}\right)^{T} \\
F=\left(f_{1}, \ldots, f_{N}, h g_{1}, \ldots, h g_{N}, h^{2} z_{1}, \ldots, h^{2} z_{N}\right)^{T}
\end{gathered}
$$


(the variables $z_{i}$ are introduced with coefficients zeros to augment the matrix $B)$,

$$
\begin{gathered}
C=\left(h y_{0}^{\prime}-\frac{3}{2} y_{0}-\frac{6127}{90720} h^{3} f_{0}-\frac{291}{30240} h^{4} g_{0}, h^{2} y_{0}^{\prime \prime}-y_{0}+\frac{99149}{272160} h^{3} f_{0}\right. \\
+\frac{725}{18144} h^{4} g_{0},-y_{0}+\frac{5}{168} h^{3} f_{0}-\frac{29}{5040} h^{4} g_{0}, 0, \ldots 0, \frac{1}{2} y_{0}+\frac{2692}{272160} h^{3} f_{0} \\
+\frac{155}{90720} h^{4} g_{0}, \frac{-1}{2} y_{0}-\frac{3475}{272160} h^{3} f_{0}-\frac{215}{90720} h^{4} g_{0}, \frac{-3}{2} y_{0}-\frac{4664}{90720} h^{3} f_{0} \\
-\frac{311}{30240} h^{4} g_{0}, 0, \ldots 0,-y_{0}-\frac{4246}{272160} h^{3} f_{0}-\frac{224}{90720} h^{4} g_{0},-y_{0}-\frac{7891}{272160} h^{3} f_{0} \\
\left.\quad-\frac{503}{90720} h^{4} g_{0},-y_{0}-\frac{13846}{90720} h^{3} f_{0}-\frac{922}{30240} h^{4} g_{0}\right) \\
L(h)=\left(\tau_{1}, \ldots, \tau_{N}, h \tau_{1}^{\prime}, \ldots, h \tau_{N}^{\prime}, h^{2} \tau_{1}^{\prime \prime}, \ldots, h^{2} \tau_{N}^{\prime \prime}\right)^{T},
\end{gathered}
$$

where $L(h)$ is the local truncation error.

$$
E=\bar{Y}-Y=\left(e_{1}, \ldots, e_{N}, h e_{1}^{\prime}, \ldots, h e_{N}^{\prime}, h^{2} e_{1}^{\prime \prime}, \ldots, h^{2} e_{N}^{\prime \prime}\right)^{T} .
$$

Theorem 3.1. Let $\bar{Y}, Y$, and $E$ be defined as above. Let $\bar{Y}$ be an approximation of the solution vector $Y$ for the system formed by combining the methods (8), (9), (10), and (11). If $e_{i}=\left|y\left(x_{i}\right)-y_{i}\right|$, he $e_{i}^{\prime}=\left|h y^{\prime}\left(x_{i}\right)-h y_{i}^{\prime}\right|$, and $h^{2} e_{i}^{\prime \prime}=\left|h^{2} y^{\prime \prime}\left(x_{i}\right)-h^{2} y_{i}^{\prime \prime}\right|$ where the exact solution $y(x)$ is several times differentiable on $[a, b]$ and if $\|E\|=\|Y-\bar{Y}\|$, then, the FDM is an eighth-order convergent method. That is $\|E\|=O\left(h^{8}\right)$.

Proof. The exact form of the system is given by (12)

$$
A Y-B F(Y)+C+L(h)=0,
$$

and the approximate form of the system is given by (13)

$$
A \bar{Y}-B F(\bar{Y})+C=0
$$

where $\bar{Y}$ is the approximation of the solution vector $Y$.

Subtracting (12) from (13) we obtain

$$
A E-B F(\bar{Y})+B F(Y)=L(h),
$$

Using the mean-value theorem, we write (14) as

$$
(A-B J) E=L(h),
$$


where the Jacobian matrix $J$ and its entries $J_{11}, J_{12}, J_{13} J_{21}, J_{22}, J_{23}, J_{31}, J_{32}$, and $J_{33}$ are defined as follows:

$$
\begin{aligned}
& J=\left[\begin{array}{ccc}
J_{11} & J_{12} & J_{13} \\
J_{21} & J_{22} & J_{23} \\
J_{31} & J_{32} & J_{33}
\end{array}\right], \quad J_{11}=\left[\begin{array}{ccc}
\frac{\partial f_{1}}{\partial y_{1}} & \cdots & \frac{\partial f_{1}}{\partial y_{N}} \\
\vdots & & \vdots \\
\frac{\partial f_{N}}{\partial y_{1}} & \cdots & \frac{\partial f_{N}}{\partial y_{N}}
\end{array}\right] \\
& J_{12}=\left[\begin{array}{ccc}
\frac{\partial f_{1}}{\partial y_{1}} & \cdots & \frac{\partial f_{1}}{\partial y_{N}} \\
\vdots & & \vdots \\
\frac{\partial f_{N}}{\partial y_{1}} & \cdots & \frac{\partial f_{N}}{\partial y_{N}}
\end{array}\right], \quad J_{13}=\left[\begin{array}{ccc}
\frac{\partial f_{1}}{\partial y_{1}} & \cdots & \frac{\partial f_{1}}{\partial y_{N}} \\
\vdots & & \vdots \\
\frac{\partial f_{N}}{\partial y_{1}} & \cdots & \frac{\partial f_{N}}{\partial y_{N}}
\end{array}\right] \text {, } \\
& J_{21}=h\left[\begin{array}{ccc}
\frac{\partial g_{1}}{\partial y_{1}} & \cdots & \frac{\partial g_{1}}{\partial y_{N}} \\
\vdots & & \vdots \\
\frac{\partial g_{N}}{\partial y_{1}} & \cdots & \frac{\partial g_{N}}{\partial y_{N}}
\end{array}\right], \quad J_{22}=h\left[\begin{array}{ccc}
\frac{\partial g_{1}}{\partial y_{1}} & \cdots & \frac{\partial g_{1}}{\partial y_{N}} \\
\vdots & & \vdots \\
\frac{\partial g_{N}}{\partial y_{1}} & \cdots & \frac{\partial g_{N}}{\partial y_{N}}
\end{array}\right] \text {, } \\
& J_{23}=h\left[\begin{array}{ccc}
\frac{\partial g_{1}}{\partial y_{1}} & \cdots & \frac{\partial g_{1}}{\partial y_{N}} \\
\vdots & & \vdots \\
\frac{\partial g_{N}}{\partial y_{1}} & \cdots & \frac{\partial g_{N}}{\partial y_{N}}
\end{array}\right], \quad J_{31}=h^{2}\left[\begin{array}{ccc}
\frac{\partial z_{1}}{\partial y_{1}} & \cdots & \frac{\partial z_{1}}{\partial y_{N}} \\
\vdots & & \vdots \\
\frac{\partial z_{N}}{\partial y_{1}} & \cdots & \frac{\partial z_{N}}{\partial y_{N}}
\end{array}\right] \\
& J_{32}=h^{2}\left[\begin{array}{ccc}
\frac{\partial z_{1}}{\partial y_{1}} & \cdots & \frac{\partial z_{1}}{\partial y_{N}} \\
\vdots & & \vdots \\
\frac{\partial z_{N}}{\partial y_{1}} & \cdots & \frac{\partial z_{N}}{\partial y_{N}}
\end{array}\right], \quad J_{33}=h^{2}\left[\begin{array}{ccc}
\frac{\partial z_{1}}{\partial y_{1}} & \cdots & \frac{\partial z_{1}}{\partial y_{N}} \\
\vdots & & \vdots \\
\frac{\partial z_{N}}{\partial y_{1}} & \cdots & \frac{\partial z_{N}}{\partial y_{N}}
\end{array}\right] .
\end{aligned}
$$

Let $M=-B J$ be a matrix of dimension $3 N$. We have

$$
(A+M) E=L(h),
$$

and for sufficiently small $h, A+M$ is a monotone matrix and thus invertible (see Jain and Aziz [5] and Jator and Li [8]). Therefore,

$$
(A+M)^{-1}=D=\left(d_{i, j}\right) \geq 0, \quad \text { and } \quad \sum_{j=1}^{3 N} d_{i, j}=O\left(h^{-3}\right) .
$$

If $\|E\|$ is the norm of maximum global error and from (15), $E=(A+$ $M)^{-1} L(h)$, using (16) and the truncation error vector $L(h)$, it follows that

$$
\|E\|=O\left(h^{8}\right) .
$$

Therefore, the TDM is an eigth-order convergent method. 


\section{Numerical Examples}

In this section, we test three linear and two non-linear numerical examples to illustrate the accuracy of the method. The global maximum absolute error is computed as $E M A X=\operatorname{Max}\left|y\left(x_{i}\right)-y_{i}\right|, i=1, \ldots, N$, where $y\left(x_{i}\right)$ is the exact solution computed at the grid point and $y_{i}$ is an approximation to the exact solution using the FDM. We note that the method requires only two function evaluations per step. All computations were carried out using our written code in Mathematica 8.0.

Example 1. Consider the linear third order BVP on $0 \leq x \leq 1$.

$$
y^{\prime \prime \prime}-x y=\left(x^{3}-2 x^{2}-5 x-3\right) e^{x}, \quad y(0)=y(1)=1, \quad \text { and } \quad y^{\prime}(0)=1
$$

The exact solution of the system is given by

$$
y(x)=x(1-x) e^{x}
$$

The FDM was tested on example 1 and results were compared with Brugnano and Trigiante (BT) (order $p=8$ ). As expected, the FDM performed better than BT.

\begin{tabular}{ccc}
\hline & FDM & BT [3] \\
$N$ & EMAX & EMAX \\
\hline 7 & $4.12 \times 10^{-12}$ & $2.89 \times 10^{-8}$ \\
14 & $1.56 \times 10^{-14}$ & $6.93 \times 10^{-11}$ \\
28 & $6.08 \times 10^{-17}$ & $1.28 \times 10^{-13}$ \\
56 & $2.37 \times 10^{-19}$ & $2.69 \times 10^{-15}$ \\
112 & $9.27 \times 10^{-22}$ & $1.448 \times 10^{-15}$ \\
& & \\
\hline
\end{tabular}

Table 1: Results for Example 1

Example 2. Consider the sandwich beam problem (linear third order) $\mathrm{BVP}$ on $0 \leq x \leq 1$ found by Krajcinovic [11] .

$$
y^{\prime \prime \prime}-l^{2} y^{\prime}+a=0, \quad y^{\prime}(0)=y^{\prime}(1)=y\left(\frac{1}{2}\right)=0
$$

The exact solution of the system is given by 


$$
\begin{aligned}
y(x)=\left(\frac{a}{l^{3}}\right)\left[\left(\sinh \left(\frac{l}{2}\right)-\sinh (l x)\right)+\right. & l\left(x-\frac{1}{2}\right) \\
+\tanh & {\left.\left[\left(\frac{l}{2}\right)\left(\cosh (l x)-\cosh \left(\frac{l}{2}\right)\right)\right]\right] }
\end{aligned}
$$

We tested the method for $\mathrm{a}=1, \mathrm{l}=5$, and 10 , and the results are compared with Brugnano and Trigiante (BT)(order $p=8$ ). It is obvious from the numerical results in Table 2 that our method performed excellently when compared with Brugnano and Trigiante method.

\begin{tabular}{ccccc}
$l=5$ & & \multicolumn{3}{c}{$l=10$} \\
\hline$N$ & FDM & BT & FDM & BT \\
\hline 14 & $5.78 \times 10^{-12}$ & $1.47 \times 10^{-9}$ & $2.26 \times 10^{-10}$ & $2.92 \times 10^{-8}$ \\
28 & $2.16 \times 10^{-14}$ & $6.70 \times 10^{-12}$ & $7.91 \times 10^{-13}$ & $2.19 \times 10^{-10}$ \\
56 & $4.94 \times 10^{-16}$ & $2.78 \times 10^{-14}$ & $2.96 \times 10^{-15}$ & $1.06 \times 10^{-12}$ \\
112 & $1.07 \times 10^{-16}$ & $1.03 \times 10^{-14}$ & $1.73 \times 10^{-17}$ & $4.21 \times 10^{-15}$ \\
& & & & \\
\hline
\end{tabular}

Table 2: Results for Example 2

Example 3. Consider the non-linear third order BVP on $0 \leq x \leq 1$.

$$
y^{\prime \prime \prime}=-2 e^{-3 y}+4(1+x)^{-3}, \quad y(0)=0, \quad y^{\prime}(0)=1, \quad y(1)=\ln 2
$$

The exact solution of the system is given by

$$
y(x)=\ln (1+x)
$$

Clearly, our results are better than those of Brugnano and Trigiante [3] . Details of the numerical results are given in Table 3.

\begin{tabular}{ccc}
\hline $\mathrm{N}$ & $\mathrm{FDM}$ & $\mathrm{BT}$ \\
\hline 7 & $5.24 \times 10^{-9}$ & $4.13 \times 10^{-7}$ \\
14 & $2.39 \times 10^{-11}$ & $5.53 \times 10^{-9}$ \\
28 & $9.50 \times 10^{-14}$ & $4.63 \times 10^{-11}$ \\
56 & $3.62 \times 10^{-16}$ & $2.16 \times 10^{-13}$ \\
112 & $2.27 \times 10^{-17}$ & $8.83 \times 10^{-16}$ \\
\hline
\end{tabular}

Table 3: Results for Example 3

Example 4. Consider the boundary-layer problem (nonlinear third order) taken from Salama [16]. 


$$
y^{\prime \prime \prime}+\alpha y y^{\prime \prime}+\beta\left(1-\left(y^{\prime}\right)^{2}\right)=0, \quad y(0)=0, \quad y^{\prime}(0)=0, \quad y^{\prime}(\infty)=1
$$

We will consider the Falkner-Skan Equation $(\alpha=1)$ and the Blasisus equations $\left(\alpha=\frac{1}{2}\right.$ and $\left.\beta=0\right)$ that are special cases of the above boundary layer problem. The results to the above solution are presented differently as there are no theoretical solutions. We looked at different values of $\beta$ (positive, zero and negative). The numerical results are shown in Table 4 for the Falkner-Skan Equation and in Table 5 for the Blasius Equation. It is clear from the table that our results are more efficient. The Number of steps needed in our method was only 10 to get the required values at the truncated boundary, where as in Brugnano and Trigiante [3], the number of steps needed was 21, and in Salama [16] $\left(\left(\varepsilon=10^{-2}\right)\right.$, the number of steps needed was 200 .

\begin{tabular}{ccccc} 
& & $F D M(N=10)$ & $\mathrm{BT}(\mathrm{N}=21)$ & Salama $(\mathrm{N}=200)$ \\
\hline$\eta_{\infty}$ & $\beta$ & $y(0)$ & $y(0)$ & $y(0)$ \\
\hline 0.93 & 40 & 7.314787 & 7.3149 & 7.314787 \\
0.95 & 30 & 6.338219 & 6.33826 & 6.338219 \\
1.13 & 20 & 5.180731 & 5.18076 & 5.180731 \\
1.49 & 10 & 3.675257 & 3.67527 & 3.675257 \\
2.57 & 2 & 1.687317 & 1.68732 & 1.687317 \\
2.88 & 1 & 1.232951 & 1.23295 & 1.2329519 \\
3.29 & 0.5 & 0.928234 & 0.928234 & 0.928234 \\
4.01 & 0 & 0.47110 & 0.471107 & 0.47110 \\
4.27 & -0.1 & 0.321838 & 0.321838 & 0.321838 \\
4.49 & -0.15 & 0.220244 & 0.220245 & 0.220244 \\
4.71 & -0.18 & 0.134948 & 0.134941 & 0.134948 \\
5.00 & -0.1988 & 0.039868 & 0.039859 & 0.039868 \\
& & & & \\
\hline
\end{tabular}

Table 4: Results for Falkner-Skan Equation for different values of $\beta$

The results of the Blasius Equation are given below:

\section{Conclusions}

We have derived a CFDM from which discrete FDMs are obtained and applied to solve $y^{\prime \prime \prime}=f\left(x, y, y^{\prime}, y^{\prime \prime}\right)$ subject to Dirichlet and Neumann boundary conditions without first adapting the ODE to an equivalent first order system. Numerical experiments are performed that show efficiency and accuracy advan- 


\begin{tabular}{ccccccc} 
FDM & $(\mathrm{N}=20)$ & & $\mathrm{BT}$ & $(\mathrm{N}=21)$ & Salama & $(\mathrm{N}=200)$ \\
\hline$\eta_{\infty}$ & $y(0)$ & $y\left(\eta_{\infty}\right)$ & $y(0)$ & $y\left(\eta_{\infty}\right)$ & $y(0)$ & $y\left(\eta_{\infty}\right)$ \\
\hline 6.67984 & 0.332151 & 4.96021 & 0.332151 & 4.96021 & 0.332 & 4.9602119 \\
8.18467 & 0.332058 & 6.4639 & 0.332055 & 6.46388 & 0.33205 & 6.4639022 \\
9.38665 & 0.332057 & 7.66586 & 0.332045 & 7.66582 & 0.3320573 & 7.6658598 \\
10.57641 & 0.332057336 & 8.85562 & 0.332057 & 8.85562 & 0.3320573366298568 & 8.8556211 \\
11.68904 & 0.332057336 & 9.96825 & 0.332057 & 9.96825 & 0.332057336629856 & 9.9682523 \\
\hline
\end{tabular}

Table 5: Results for different values of the truncated boundary $\eta_{\infty}$ for Blasius Equation

tages of the method over existing ones in the literature. Details of the numerical results are displayed in Tables $1,2,3,4,5,6$.

\section{References}

[1] K. E. Atkinson, An introduction to numerical analysis, 2nd edition John Wiley and Sons, New York (1989).

[2] D. O. Awoyemi, A P-stable linear multistep method for solving general third order ordinary differential equations, Intern. J. Comput. Math, 80, (8) (2003), 987-993.

[3] L. Brugnano and D. Trigiante, Solving Differential Problems by Multitep Initial and Boundary Value Methods, Gordon and Breach Science Publishers, Amsterdam, (1998), 280-299.

[4] P. Henrici, Discrete Variable Methods for ODEs, John Wiley, New York, USA (1962).

[5] M. K. Jain and T. Aziz, Cubic spline solution of two-point boundary Value with signifigant first derivatives, Comp. Meth. Appl. Mech. Eng., 39 (1983), pp. 83-91

[6] S. N. Jator, Multiple Finite Difference Methods for Solving Third Order Ordinary Differential Equations, International Journal of Pure and Applied Mathematics, 43, (2), (2007), 253-265.

[7] S. N. Jator, Novel Finite Difference Schemes for Third Order Boundary Value Problems, Intern. J. Pure and Appl. Math., 53(2009), 37-54. 
[8] S. N. Jator and J. Li, An algorithm for second order initial and boundary value problems with an automatic error estimate based on a third derivative method, Numerical Algorithms, 59, (2012), 333-346.

[9] S.N. Jator, S. Swindle, and R. French, Trigonometrically fitted block numerov type method for $y^{\prime \prime}=f\left(x, y, y^{\prime}\right)$, published online in Numer Algor., (2012).

[10] H. B. Keller, Numerical methods for two-point boundary value problems, Blaisdell, Waltham, Mass.; 184 pp. QA372.K42 (1968).

[11] D. Krajcinivic, Sandwich Beam Analysis, Journal of Applied Mechanics 39 (1972) 773-778.

[12] A. Khan and T. Aziz, The Numerical Solution of Third-Order BoundaryValue Problems Using Quintic Splines, Appl. Math. Comput., vol. 137, (2003), 253-260.

[13] I. Lie and S. P. Norsett, Superconvergence for Multistep Collocation, Math Comp. 52, (1989), $65-79$.

[14] P. Onumanyi, S. N. Jator and U. W. Sirisena, Continuous finite difference approximations for solving differential equations, Inter. J. Compt. Maths. 72 , (1) (1999), 15-27.

[15] P. Onumanyi, D. O. Awoyemi, S. N. Jator and U. W. Sirisena, New linear mutlistep methods with continuous coefficients for first order initial value problems, J. Nig. Math. Soc. 13, (7) (1994), 37-51.

[16] A.A. Salama, Higher-Order Method For Solving Free Boundary Value Problems, Numerical Heat Transfer, Part B: Fundamentals:An International Journal of Computation and Methodology 45 (2004) 385-394.

[17] A. A. Salama and A. A. Mansour, Fourth-Order Finite Diffence method for Third-order Boundary-value Problems, Numerical Heat Transfer, Part B, 47, (2005), 383-401.

[18] L.F. Shampine, S. Thompson, Scholarpedia, 2, No. 3 (2007), 2861.

[19] Siraj-Ul-Islam and I. A. Tirmizi, A smooth approximation for the solution of special non-linear third-order boundary-value problems based on nonpolynomial splines, International Journal of Computer Mathematics, 83, (4), (2006), 397-407. 
[20] A. Tirmizi, E.H. Twizell, Siraj-Ul-Islam, A numerical method for thirdorder non-linear boundary-value problems in engineering, Intern. J. Computer Math. 82 (2005) 103-109.

[21] E.O. Tuck, L.W. Schwartz, A numerical and asymptotic study of some third order ordinary differential equation relevant to draining and coating flows, SIAM Review, 32, No. 3 (1990), 453-549.

[22] S. Valarmathi, N. Ramanujam, Boundary value technique for finding numerical solution to boundary value problems for third order singular perturbed ordinary differential equations, Intern. J. Computer Math., 79, No. 6 (2002), 747-763. 
